\title{
Mediastinum Drainage by Cervicotomy in the Neck and Mediastinum Cellulitis: About 12 Cases
}

\author{
El Ansari R*, Tatari MM, Lahjaouj M, Abada RL, Rouadi S, Roubal M and Mahtar M \\ Department of ENT, 20 Auguste 1953 Hospital in Casablanca, Morocco
}

*Corresponding author: El Ansari R, Intern, Department of ENT, 20 Auguste 1953 Hospital in Casablanca, Morocco, E-mail: dr.elansari66@gmail.com

Citation: El Ansari R, Tatari MM, Lahjaouj M, Abada RL, Rouadi S, et al. (2016) Mediastinum Drainage by Cervicotomy in the Neck and Mediastinum Cellulitis: About 12 Cases. J Case Rep Stud 5(1): 102. doi: 10.15744/2348-9820.5.102

Received Date: November 09, 2016 Accepted Date: February 24, 2017 Published Date: February 27, 2017

\begin{abstract}
Mediastinum cellulitis is a serious ENT emergency of significant mortality. The prognosis depends on the period of care and diagnostic. The management sometimes requires the cooperation ENT, thoracic Surgery and reanimation.

It is a retrospective study about 12 cases presenting neck and mediastinum cellulitis treated at the ENT department, 20 August 1953 Hospital in Casablanca Morocco, spreading over 3 years (September 2012-septembre 2015).

We have 70 cases of cervicofacial cellulitis during this 3 years, only 12 of them are retained who presenting mediastinum complications. It is very important to search utilisation of non-steroid anti-inflammatory drugs or cortisone medications during inflammatory episode, and unbalanced diabetes.

All the patients received the same management scheme and stayed in intensive care unit depending on the degrees of severity.

We obtain favourable evolution especially after stabilization of tare specially diabetes which remains the most common cause.

Two daily dressing changes with extensive washing with hydrogen peroxide and povidone-iodine has proven effective.

Keywords: Mediastinum cellulitis; ENT; Cervicotomy
\end{abstract}

\section{Introduction}

Cellulitis remains a very serious disease even today. It's a medical and surgical emergency and can threaten patient's health and life because of risk of developing descending necrotizing mediastinitis. Mortality, which varied between 10-40\%, has been reduced owing to the standard securing of airway patency and use of an appropriate surgical treatment approach. The management sometimes requires the cooperation ENT and Thoracic Surgery. Patients carried out drainage by cervicotomy with extensive washing by povidone-iodine have good results comparing with patient having thoracotomy because of the high risk of morbidity. That is what we describe in this study.

\section{Materials and Methods}

It is a retrospective study of a total of 12 patients with the diagnosis of neck and mediastinum cellulitis was treated at the ENT Unit, 20 August 1953 Hospital in Casablanca Morocco, during 3 years (September 2012- September 2015).

We used the admission records of emergency unit and the medical files.

70 cases of cervicofacial cellulitis were harvested from our registers, but only the complicated cellulitis of mediastinitis was retained.

Patients received intravenous injection of antibiotics: amoxicillin/ Clav. Acid + metronidazole+ aminoacid quickly adjust based on the bacteriological culture analysis. We found commonly the streptococcus anaerobius and the fusobacterium in the bacteriological analysis.

The surgery is always practiced by cervicotomy and drainage, excision of necrotic tissue, extensive washing by povidone-iodine, drainage by setting Delbet blade, flap reconstruction planned for patients presenting large skin necrosis. All patients carried out cervicotomy and no thoracotomy has done (Figure 1).

Patients were convoked 2 months after they leave the department for control with Neck and thoracic CT scan. All of them come without recidivism or complications. 


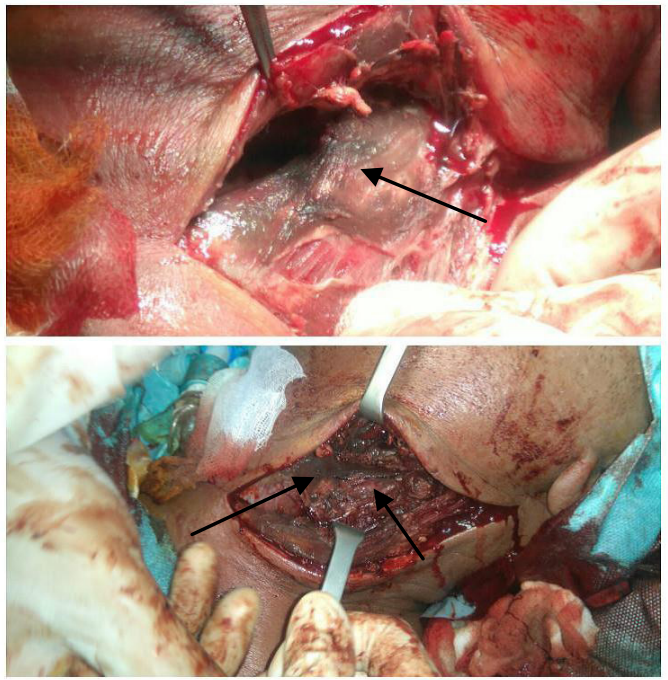

Figure 1: Cervicotomy and drainage of the neck and mediastinum cellulitis of one of the 12 cases $(\rightarrow$ : anterior board of Sternocleido mastoid muscle with pus issue)

\section{Results}

The average age varies depending on the origin of infection and the field of the patients ranging between 20 and 60 years, so 40 years. The sex ratio is 1,33 with majority of male.

The origin was principally odontogenic (9 patients) or untreated tonsillitis (3 cases).

The principal causes are diabetes ( 8 cases) prolonged use of cortisone ( 2 cases), and non- steroid anti-inflammatory drugs (NSAIDs) (2 cases).

Clinical signs is painful inflammatory swelling of the neck (97\%), trismus (95\%), fever (54\%), dysphagia (27, 27\%), and skin necrosis $(18,18 \%)$.

CT scan cervicothoracic (Figure 2) with injection of contrast product is performed in all patients. It has an interest in the study of locoregional extension, the origin of infection, the surgical approaches and the complications of cellulitis.

The treatment is medical based of broad-spectrum antibiotics and surgical depending of the site of infection.

All patients stayed in intensive care unit for period going between 5 days to 3 weeks.

We obtain favourable evolution especially after stabilization of diabetes.

Neck and thoracic CT scan was done after 2 Months showing the total resorption of neck and mediastinum collection (Figure 3).

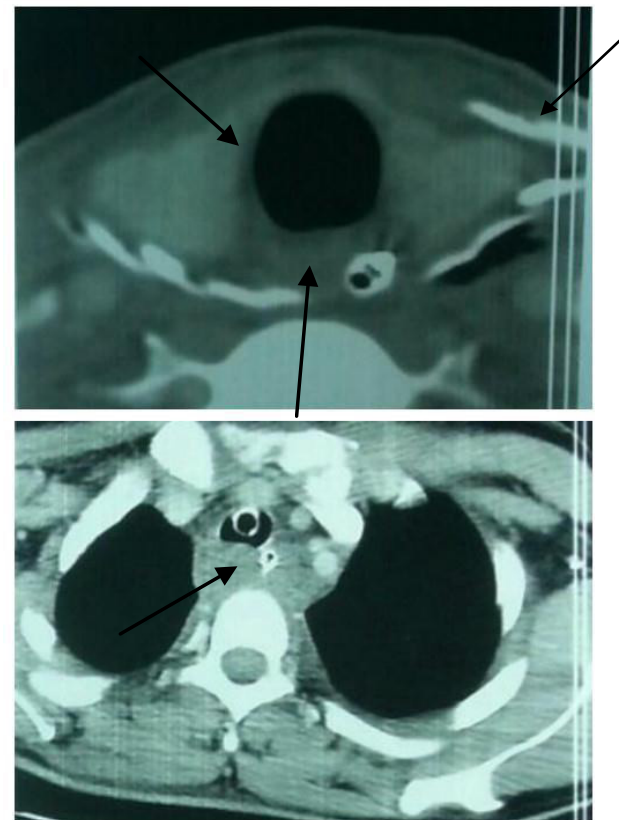

Figures 2: CT scan of neck and thorax showing neck and mediastinum collection $(\rightarrow)$ (CT scan of one of the 12 cases) 

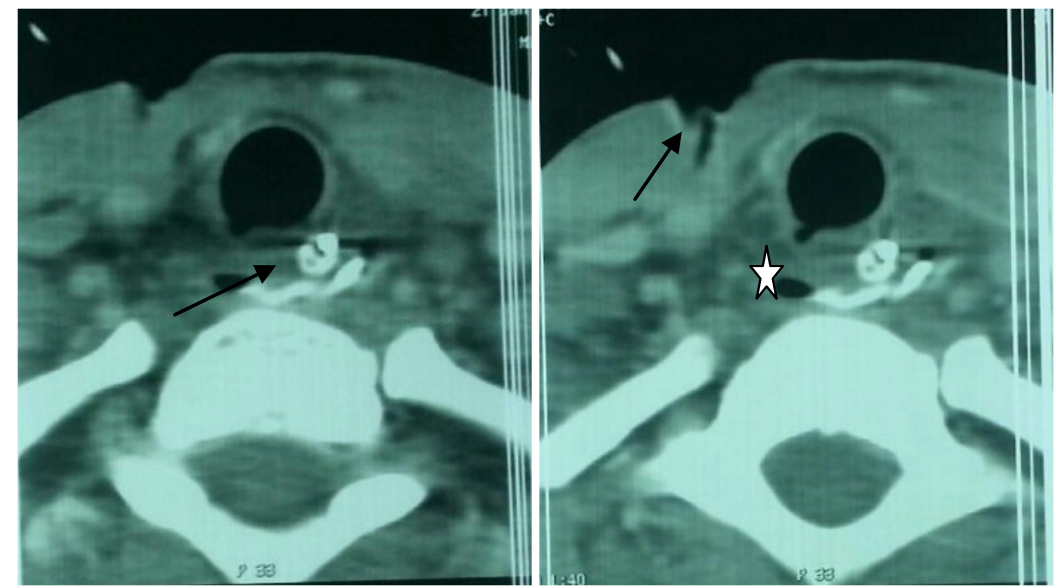

Figure 3: Control neck and thoracic CT scan after 2 mounths $(\rightarrow$ Medium mediastinum without collection recidism, $\hat{\omega}$ persistent air bubble after collection drainage)

\section{Discussion}

Odontogenic and oropharyngeal infections are relatively common in the cervicocephalic region. Often, early diagnosis and immediate antibiotic treatment or surgical drainage contains the infection, allowing its resolution. In rare cases, odontogenic or peritonsillar abscesses may spread through the deep fascial cervical spaces, producing life-threatening complications, especially when they reach the mediastinum.

Described for the first time by Pearse [1] in 1938, Descending necrotizing mediastinitis (DNM) is a rare [2], acute polymicrobic infection of the mediastinum that develops rapidly after an odontogenic or oropharyngeal infection. The responsible pathogens are mainly aerobic and anaerobic bacteria of the oralmicroflora. Estrera et al. [3] and Makeieff et al. [4] report a prevalence of Streptococcus anaerobius, Bacteroides, and Fusobacterium, which are found commonly in the upper digestive and respiratory tracts and are not virulent normally, that what we found in our study.

Local and general body defense mechanisms are usually sufficient to contain the bacterial proliferation, except in certain virulent strains or when the patient's general health is poor.

Anaerobes have an especially high affinity for the lipid constituents of cellular membranes, leading to the hydrolysis of muscle cells, erythrocytes, and platelets. Their virulence increases when the oxygen pressure in tissues decreases, producing enzymes such as fibrinolysin and coagulase, which result in ischemia at the infected site and favour bacterial proliferation. Other enzymes, such as hyaluronidases, proteases, and collagenases, distort fundamental tissue constituents (collagen), split the support structures, and facilitate the spread of infections along the cervical fascia. Therefore, any pathologic state that lowers tissue oxygenation, including diabetes, connective tissue disorders, and acquired immunodeficiency, can favour the spread of infection.

The infection then spreads along the cervicomediastinal fascial spaces and planes along the superficial, visceral, and prevertebral layers of the deep cervical fascia, which create the pretracheal, previsceral, or vascular, and retrovisceral or prevertebral spaces, respectively, in the neck. In each of these potential spaces, loose areolar tissue, which lacks defense cells and is poorly vascularized, is connected with the pericardium, pleura, and mediastinum, serving as a portal of entry for an oropharyngeal or odontogenic infection into the chest. Oropharyngeal infections spread mainly into the retrovisceral space to reach the posterior mediastinum, whereas odontogenic submental and submandibular abscesses or phlegmons tend to spread posteriorly toward the vascular space and then to the anterior mediastinum.

Patients who are suspected of having DNM must be managed immediately; a delay in diagnosis is one of the main reasons for the high mortality rate in DNM. The diagnosis of cervical infection is obvious clinically, but the early diagnosis of mediastinitis is often difficult because of the vagueness of early symptoms implicating mediastinal involvement and the use of non-steroid anti-inflammatory drugs (NSAIDs) that reduce functional symptoms. The patient suffers chest pain, dyspnea, and fever, with significant associated toxemia.

Although NSAIDs cannot be incriminated in CFC (cervicofacial cellulitis) with absolute certainty, several series, including the present, tend to implicate them, if not as a direct causal factor then at least as promoting or aggravating CFC. Mathieu et al. [5], in a series of 45 patients admitted with CFC, found NSAID use in $44 \%$ of cases. Merle et al. [6] highlighted NSAIDs as promoting CFC, reporting a $76.46 \%$ NSAID rate, similar to the present findings but in a smaller series of only 17 patients presenting with odontogenic CFC; 8 presented mediastinitis, 4 of whom had taken NSAIDs, and 7 of whom died. In a larger series of 94 CFC patients admitted to intensive care between 1995 and 2005, Shaikh et al. reported NSAID use in 80\% of patients [7]. An association between cellulitis complicated by odontogenic mediastinitis and NSAID use was reported in several other studies [68]. Cellulitis other than cervicofacial has also been reported, with NSAIDs implicated in evolution toward fatal necrotizing fasciitis 
[9]. This correlation may be explained by the action mechanism of NSAIDs against inflammation, which is after all basically a non-specific defense mechanism against microbial invasion. NSAIDs inhibit the degradation of cellular arachidonic acid by the cyclo oxygenase pathway, preventing production of thrombox-ane A2 and prostaglandin, which play an important role in cellular chemotaxis. NSAIDs thus oppose polynucleate cell and macrophage migration and phagocytosis. They also reduce the early signs of inflammation, thus delaying consultation [10,11]. The harmful impact of NSAIDs in infection was borne out by experimental studies, including that by Solberg et al. On phenylbutazone, demonstrating impaired granulocyte activation, phagocytosis and intracellular destruction of streptococci and staphylococci in vitro [12]. However, while the clinical and in vitro role of NSAIDs in aggravating infection has been often reported [10], some animal studies found no significant difference between experimental groups with CFC taking diclofenac versus placebo [10,11]. However, these studies had very small samples (some 20 individuals per arm), and the origin of infection was subcutaneous injection, as opposed to odontogenic or oropharyngeal in the great majority of human series. The animal models fail to fully reproduce the anatomic and bacteriological conditions found as CFC risk factors in humans. Even so, other animal studies confirmed the role of NSAIDs in aggravating cellular tissue infection [13].

All patients underwent cervical and thoracic CT scan, with injection of contrast product, so only thoracic CT or cervicothoracic CT confirms the diagnosis of mediastinitis immediately with high accuracy, showing soft tissue infiltration with loss of the normal fat planes or collections of fluid with or without gas bubbles. Furthermore, cervicothoracic CT shows the continuity of the infection process between the neck and chest, establishing a relationship between a neck infection and mediastinitis.

DNM should be treated as a medical and surgical emergency. In addition to surgery, empirical high dose intravenous antibiotics should be initiated immediately with penicillin G $2.4 \mathrm{~g}$ daily in 4 divided doses, combined with metronidazole 500 mg and gentamicin $80 \mathrm{mg}$, both every 8 hours. Moreover, patients known to be immunocompromised may require other antibiotics, such as piperacillin or imipenem [14]. The antibiotic therapy can be modified subsequently based on culture results and antibiotic susceptibility. The debate over the use of steroids in the management of serious head and neck infections of the upper respiratory tract is ongoing. The controversy stems from the anti-inflammatory and immunosuppressant actions of steroids. Recent studies have recommended intravenous dexamethasone $10 \mathrm{mg}$ as an initial dose, followed by 4 mg every 6 hours for 48 hours; the antiinflammatory effect of dexamethasone resolves the edema and cellulitis, providing chemical decompression, protecting the airways, and allowing better antibiotic penetration into the area [15-17]. At the same time, wide unbridling and evacuation of suppurated cervicothoracic collections should be carried out urgently, with extensive excision of necrotic tissue to limit the spread of infection and decrease the morbidity and mortality.

Our patients underwent cervicotomy for mediastinum cellulitis with extensive washing for two time per day until disappearance of clinical inflammatory signs and cervicothoracic CT scan control. Several approaches are described for the optimal transthoracic approach, including the subxiphoid approach, clamshell incision, median sternotomy, and thoracoscopic approach. Although each of these techniques has potential advantages and disadvantages, the posterolateral thoracotomy incision remains the standard $[1,18-20]$. It allows comprehensive access to a hemithorax, including the ipsilateral mediastinum and pericardium, and provides the broadest exposure of the prevertebral and paraesophageal planes without the risk of sternal osteomyelitis, which may follow a sternotomy or clamshell incision.

The cervical approach alone does not allow sufficient drainage in cases of serious inferior mediastinal extension. Estrera et al. [3] showed the cervical approach to the mediastinum only in cases where thoracic CT showed limited extension of the infiltration above the tracheal bifurcation anteriorly and the fourth thoracic vertebra posteriorly.

The need for a routine intraoperative tracheotomy warrants a separate discussion. According to some authors, a tracheotomy should be included as part of the therapeutic strategy, because patients often require prolonged postoperative assisted ventilation, or because there is a likelihood that they will develop an upper airway obstruction due to a lateral cervical abscess or pharyngeal edem $[3,21,22]$. In this case 9 patients carried out tracheotomy because of the inspiratory dyspnea they presented in the admission in the emergency.

\section{Conclusion}

Neck and mediastinum cellulitis is rare but need urgent care. Odontogenic and oropharyngeal infections are relatively common. It can threaten patient's health and life because of risk of developing descending necrotizing mediastinitis especially in the diabetic's patients and patients under cortisone or NSAIDs for long time. It should be treated as a medical and surgical emergency.

Patients carried out drainage by cervicotomy with extensive washing by povidone-iodine have good results comparing with patient having thoracotomy because of the high risk of morbidity. Diabetes or others causes must be detected and treated to have good results.

\section{References}

1. Pearse HE Jr (1938) Mediastinitis following cervical suppuration. Ann Surg 107: 588-611.

2. Sancho LM, Minamoto H, Fernandez A, Sennes LU, Jatene FB (1999) Descending necrotizing mediastinitis: A retrospective surgical experience. Eur J Cardiothorac Surg 16: 200-5. 
3. Estrera AS, Landay MJ, Grisham JM, Sinn DP, Platt MR (1983) Descending necrotizing mediastinitis. Surg Gynecol Obstet 157: 545-52.

4. Makeieff M, Gresillon N, Berthet JP, Garrel R, Crampette L, et al. (2004) Management of descending necrotizing mediastinitis. Laryngoscope 114: 772-5.

5. Mathieu D, Neviere R, Teillon C, Chagnon JL, Lebleu N, et al. (1995) Cervical necrotizing fasciitis: clinical manifestations and management. Clin Infect Dis 21: 51-6.

6. Merle JC, Guerrini P, Beydon L, Margenet A, Tchakerian A, et al. (1995) Cellulites cervico faciales odontogéniques. Eur J Emerg 8: $14-9$.

7. Shaikh N, Ummunissa F, Hanssen Y, Al Makki H, Shokr HM (2010) Hospital epidemiology of emergent cervical necrotizing fasciitis. J Emerg Trauma Shock 3: 123-5.

8. Demeslay J, Bonnecaze G, Vairel B, Chaput B, Pessey JJ, et al. (2014) Implications of anti-inflammatory in the complications of pharyngitis. A retrospective analysis of 163 cases [Impli-cations des anti-inflammatoires dans les complications des pharyngites. Uneanalyse rétrospective de 163 cas] Ann Fr Otorhinolaryngol Pathol Cervicofac 131: 286-90.

9. Verfaillie G, Knape S, Corne L (2002) A case of fatal necrotizing fasciitis after intra-muscular administration of diclofenac. Eur J Emerg Med 9: 270-3.

10. Eter EG, Khazzaka A, Mneimneh W, Karam-Sarkis D, Haddad A, et al. (2009) Does diclofenac increase the risk of cervical necrotizing fasciitis in a rat model? Int J Exp Pathol 90: 58-65.

11. Guibal F, Muffat-Joly M, Terris B, Garry L, Morel P, et al. (1998) Effects of diclofenac on experimental streptococcal necrotizing fasciitis (NF) in rabbit. Arch Dermatol Res 290: 628-33.

12. Solberg CO, Allred CD, Hill HR (1978) Influence of phenylbutazone on leuko-cyte chemiluminescence and function. Acta Pathol Microbiol Scand C 86C: 165-71.

13. Weng TC, Chen CC, Toh HS, Tang HJ (2011) Ibuprofen worsens Streptococcus pyogenes soft tissue infections in mice. J Microbiol Immunol Infect 44: 418-23.

14. Chow AW (1992) Life-threatening infections of head and neck. Clin Infect Dis 14: 991-1004.

15. Busch RF, Shah D (1997) Ludwig's angina: improved treatment. Otolaryngol Head Neck Surg 117: S172-5.

16. Srirompotong S, Art-Smart T (2003) Ludwig's angina: a clinical review. Eur Arch Otorhinolaryngol 260: 401-3.

17. Ridder GJ, Technau-Ihling K, Sander A, Boedeker CC (2005) Spectrum and management of deep neck space infections: An 8-year experience of 234 cases. Otolaryngol Head Neck Surg 133: 709-14.

18. Marty-Ané CH, Berthet JP, Alric P, Pegis JD, Rouvière P, et al. (1999) Management of descending necrotizing mediastinitis: An aggressive treatment for an aggressive disease. Ann Thorac Surg 68: 212-7.

19. Freeman RK, Vallières E, Verrier ED, Karmy-Jones R, Wood DE (2000) Descending necrotizing mediastinitis: An analysis of the effects of serial surgical debridement on patient mortality. J Thorac Cardiovasc Surg 119: 260-7.

20. Papalia E, Rena O, Oliaro A, Cavallo A, Giobbe R, et al. (2001) Descending necrotizing mediastinitis: surgical management. Eur J Cardiothorac Surg 20: 739-42.

21. Cordero L, Torre W, Freire D (1996) Descending necrotizing mediastinitis and respiratory distress syndrome treated by aggressive surgical treatment. J Cardiovasc Surg 37: 87-8.

22. Wheatley MJ, Stirling MC, Kirsh MM, Gago O, Orringer MB (1990) Descending necrotizing mediastinitis: transcervical drainage is not enough. Ann Thorac Surg 49: 780-4. 